\title{
THE EFFICACY OF PLATELET RICH PLASMA IN THE TREATMENT OF PERIARTHRITIS OF SHOULDER JOINT IN DIABETICS VS NON-DIABETICS
}

\author{
Dr. T. Venugopal Junior Resident $-3^{\text {rd }}$ year \\ Dr. (Col.) M.E. \\ Luther* \\ Professor of Orthopaedics ${ }^{\star}$ Corresponding Author \\ Dr. Y. Bhanurekha Prof and HOD, Department of Orthopedics
}

\section{KEYWORDS :}

\section{INTRODUCTION :}

Periarthritis of shoulder joint / adhesive capsulitis also commonly known as Frozen shoulder, occurs due to adhesion at the glenohumeral joint ${ }^{1}$. The term "Frozen Shoulder" was first described in 1934 by Codman. ${ }^{2}$ The incidence of periarthritis of shoulder joint is around $3-5 \%$ in the general population ${ }^{3}$. It is more common in females ${ }^{4}$ and develops between the ages of 40 to 70 years ${ }^{3}$. The main cause of painful restriction of movement in frozen shoulder is an inflammatory contracture of the joint capsule ${ }^{5}$. A meta-analysis showed that patients with diabetes were 5 times more likely than nondiabetics to have adhesive capsulitis. Also, the overall prevalence of adhesive capsulitis in diabetics was estimated at $13.4 \%{ }^{6}$. According to a population-based followup study, patients with diabetes had an increased risk of developing adhesive capsulitis of shoulder joint as compared to nondiabetics ${ }^{7}$. The increased incidence of adhesive capsulitis in diabetics might be due to the glycosylation of the collagen within the shoulder joint triggered by the presence of high blood sugar ${ }^{8}$.

\section{AIM AND OBJECTIVES :}

- To study the efficacy of platelet rich plasma in the treatment of periarthritis shoulder in diabetics and nondiabetics.

- To compare the degree of improvement in function and quality of life in both diabetics and non-diabetic patients of periarthritis of shoulder who were treated with Platelet Rich Plasma.

\section{MATERIALS AND METHODS :}

A Prospective observational study was conducted from October 2019 to September 2020. A total of 30 patients were enrolled in study, of which 19 were diabetic and 11 were nondiabetic. Patients presenting to the OPD with shoulder pain and fulfilling the inclusion and exclusion criteria were enrolled in the study. In the first visit, before $1^{\text {st }}$ dose of PRP [Platlet Rich Plasma] injection the patient was assessed using objective assessment tools - VAS [Visual Analogue Scale] \& SPADI [Shoulder Pain And Disability Index] and given the $1^{\text {st }}$ dose of PRP [Platlet Rich Plasma]. The patients were advised to continue physiotherapy after the PRP treatment. On the second visit ,2 weeks after $1^{\text {st }}$ intra-articular injection, the patient's Visual Analogue Scale and Shoulder Pain And Disability Index were assessed and a second dose of PRP was given. On the third visit [after 6 weeks from $1^{\text {st }}$ injection of PRP] patients Visual Analogue Scale score and Shoulder Pain And Disability Index score were assessed.

\section{INCLUSION CRITERIA :}

- Age more than 35 years.

- Patients with normal ESR, CRP and platelet count .

- Shoulder pain for at least one month associated with more than one-third loss of active shoulder flexion, abduction and external rotation.

- Normal antero-posterior radiographs of the glenohumeral joint in neutral rotation.

\section{EXCLUSION CRITERIA :}

- Age less than 35 years.

- Intrinsic glenohumeral pathology.

- History of shoulder trauma/surgery.

- Clinical evidence of complex regional pain syndrome.

- History of steroid injection in the involved shoulder joint during the preceding six months or non-steroidal antiinflammatory drugs intake in the last seven days

- Patients with hematological disorders or on antiplatelet or anticoagulant therapy.

After obtaining a written informed consent, a detailed history was taken regarding the duration of pain, type of movements restricted, any disruption of sleep / daily activities due to pain. Relevant past medical history was also obtained. The following investigations were done during the first visit and patients with normal parameters were included in the study:

\section{ESR, CRP, CBP,}

2. BT, CT

3. FBS, PLBS, $\mathrm{HbAlC}$

4. X-ray shoulder - antero posterior view and axillary view.

Known diabetics with controlled sugar levels were also included in the study.

PRP PREPARATION : PRP will be obtained from a sample of patient's blood. $20 \mathrm{ml}$ venous blood will be drawn to yield $5 \mathrm{ml}$ of PRP. Citrate dextrose A (an anticoagulant) will be added. Preparation of PRP will be done by double centrifugation process. After the first centrifugation process (at 1500rpm for 5 $\mathrm{min})$, three layers will be obtained:

\section{Plasma (superior layer) \\ 2. Buffy coat (platelets with leukocytes) \\ 3. Erythrocytes (inferior layer)}

The first and second layers will be transferred to an empty tube and centrifuged (at 3000 rpm for 10) again. Three layers will be obtained. The first layer will be discarded. The second layer $(\sim 10 \mathrm{ml})$ is platelet rich plasma which is used for intracapsular injection into the shoulder joint. The whole process will be repeated after 2 weeks for the second dose of PRP.

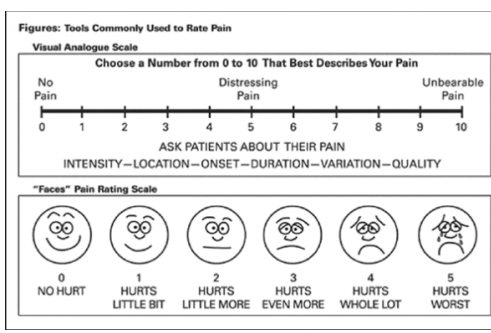




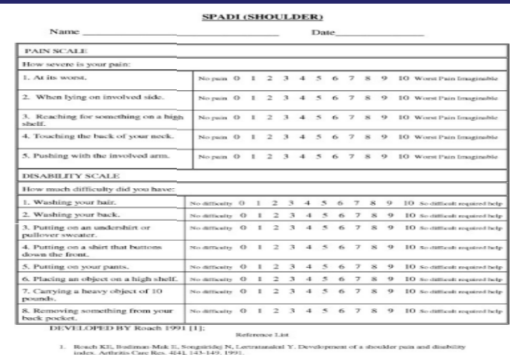

TABLE NO 1 : DISTRIBUTION OF CASES BASED ON GENDER AND DIABETES WITHIN THE PRESENT STUDY $(n=30)$

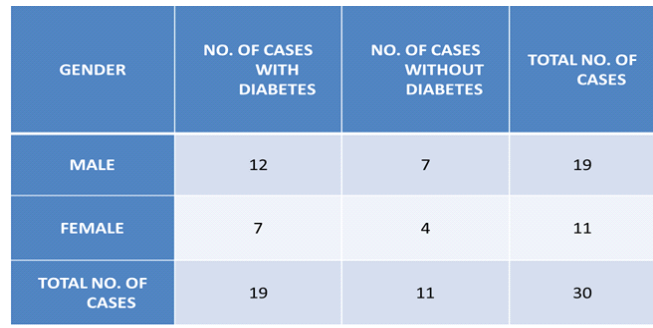

A majority of the subjects were a known case of diabetes.

GRAPH NO 1 : DISTRIBUTION OF CASES BASED ON GENDER AND DIABETES WITHIN THE PRESENT STUDY $(\mathrm{n}=30)$

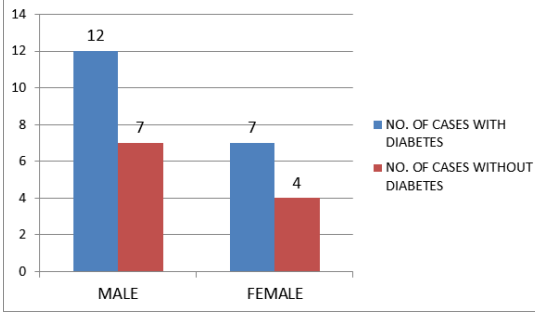

In the present study which has a total of 19 males; 12 of them had diabetes, while out of 11 females, 7 of them had diabetes.

\section{TABLE NO 2 : MEAN SPADI AMONG DIABETICS AND NON DIABETICS IN ALL 3 VISITS $(n=30)$.}

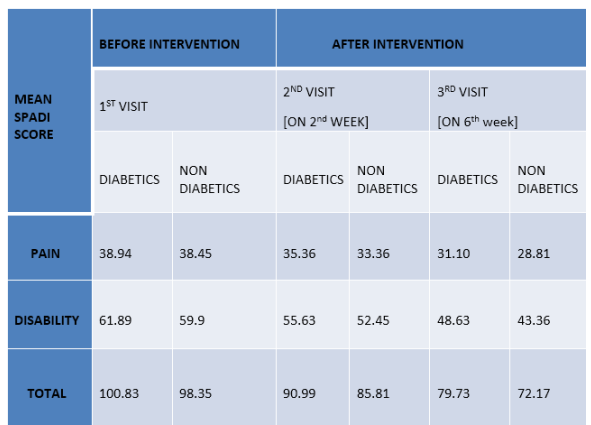

GRAPH NO 2 : MEAN SPADI AMONG DIABETICS AND NON DIABETICS IN ALL 3 VISITS $(n=30)$

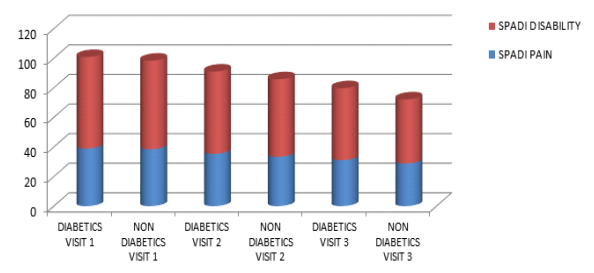

Although there is a progressive decline in SPADI among both diabetics and non diabetics in the $2^{\text {nd }}$ and ${ }^{3 r d}$ visits when compared to the first visit; this difference is more pronounced in non diabetics.
TABLE NO 3 : MEAN VISUAL ANALOGUE SCALE AMONG DIABETICS AND NON DIABETICS IN ALL 3 VISITS $(n=30)$

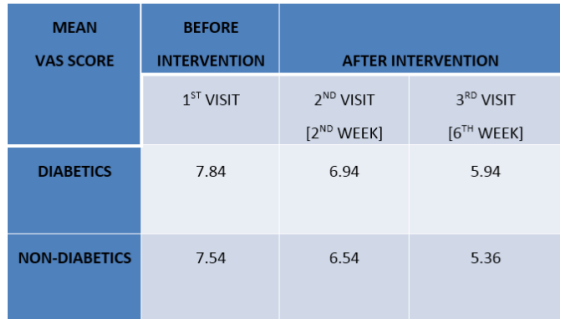

GRAPH NO 3 : MEAN VISUAL ANALOGUE SCALE AMONG DIABETICS AND NON DIABETICS IN ALL 3 VISITS $(n=30)$

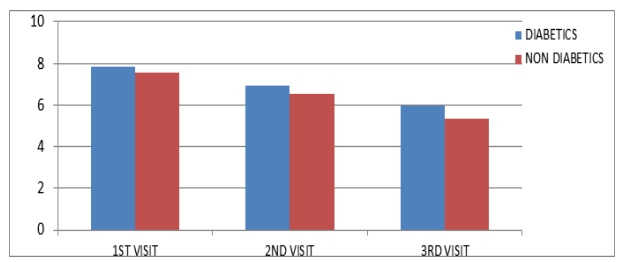

There is a good decline in VAS among both diabetics and non diabetics in the $2^{\text {nd }}$ and $3^{\text {rd }}$ visits when compared to the $1^{\text {st }}$ visit, but the decline is more in non-diabetics as compared to diabetics.

\section{DISCUSSION :}

In the present study, we tried to evaluate the efficacy of Platelet rich Plasma injection in the treatment of periarthritis of shoulder joint in diabetics and non-diabetics. The current modalities used for the treatment of periarthritis of shoulder joint like physiotherapy, corticosteroid injection are not ideal due to time taken for results and increased incidence of complications. Surgical methods are invasive and may not always bring about the desired result.

Thus arises the need to develop a novel treatment approach for periarthritis of shoulder joint. PRP is affordable, safe and easily available since it is derived from the patient's own blood. PRP works through growth factors which play a key role in rehabilitation of injuries and diseases. Growth factors stimulate repair and healing of tendons, ligaments and cartilage, epithelialization and formation of new connective tissue. Various studies have been conducted that prove the efficacy of PRP in the treatment of many injuries like refractory jumper's knee, lateral epicondylitis etc ${ }^{9,10}$.

In the current study, the mean SPADI among diabetics during the $1^{\text {st }}, 2^{\text {nd }}$ and $3^{\text {rd }}$ visit was $100.83,90.99$, and 79.93 respectively, whereas in non-diabetics the score was $98.35,85.81$, and 72.17 respectively. The mean VAS among diabetics during the $1^{\text {st }}, 2^{\text {nd }}$ and $3^{\text {rd }}$ visit was $7.84,6.94$ and 5.94 respectively whereas in non-diabetics the score was $7.54,6.54$ and 5.36 respectively. Although the presentation of adhesive capsulitis may be the same in diabetics and non diabetics ${ }^{11}$, the incidence of adhesive capsulitis is more in diabetics as compared to nondiabetics.

The study shows that although PRP is effective in the treatment of adhesive capsulitis, the efficacy is more in non-diabetics.

\section{CONCLUSION :}

This study has conclusively proved that the improvement in pain and range of motion (assessed via SPADI \& VAS) is more in non-diabetics as compared to diabetics in patients of periarthritis of shoulder joint who were treated with Platelet Rich Plasma.

\section{REFERENCES :}

1. M. Jeyaraman, Ramesh R., Prajwal G. S., H. J. Dhamsania. The comparative and prospective study on efficacy and functional outcome of autologous platelet rich plasma injection vs hydrodissection in adhesive capsulitis of 
shoulder. International Journal of Research in Orthopedics. 2018 Nov;4(6):848-853

2. H. Aslani, S. T. Nourbakhsh, Z Zafarani, M. A Bani, M. E. S. Ananloo, M. Beigy, S. Salehi. Platelet rich plasma for frozen shoulder : A case report. Archives of bone and joint surgery. 2016;4(1):90-93.

3. S. Y. Kothari, V. Srikumar, N. Singh. Comparative Efficacy of Platelet Rich Plasma Injection, Corticosteroid Injection and Ultrasonic Therapy in the Treatment of Periarthritis Shoulder. Journal of Clinical and Diagnostic Research. 2017 May, 11(5): RC15-RC18.

4. Alok Chandra Agrawal, Buddhadeb Nayak, Harshal Sakale. Management of Adhesive Capsulitis of Shoulder Joint by Single Platelet Rich Plasma Injection. Journal of orthopaedics, traumatology and rehabilitation. 2019; 11(1):62-5

5. K Tamai, M. Akutsu, Y. Yano. Primary frozen shoulder: brief review of pathology and imaging abnormalities. Journal of Orthopaedic Science. 2014; 19:1-5

6. N. H. Zreik, R. A. Malik, C. P. Charalambous. Adhesive capsulitis of the shoulder and diabetes : a meta-analysis of prevalence. Muscles, Ligaments and Tendons Journal 2016:6 (1):26-34.

7. Y.P. Huang, C. Y. Fann, Y. H. Chiu, M. F. Yen, L. S. Chen, H. H. Chen, S. L. Pan. Associationof diabetes mellitus with the risk of developing adhesive capsulitis of shoulder : A longitudinal population based follow up study. Arthritis care and research. 2013;65(7):1197-1202.

8. J. Macgillvray. M. Drakos. Practical Diabetology. June 2001.

9. G. Filardo, E.Kon, S. Della Villa, F. Vincentelli, P. M. Fornasari, M. Marcacci. Use of platelet rich plasma for the treatment of refractory jumper's knee. International Orthopaedics (SICOT). 2010; 34:909-915.

10. J. C. Peerbooms, J. Sluimer, D. J. Bruijin, T. Gosens. Positive effect of autologous platelet concentrate in lateral epicondylitis in a double - blind randomized controlled trial : platelet rich plasma versus corticosteroid injection with a l-year follow-up. The American Journal of Sports Medicine. 2010;38(2):255-262

11. M. M. Uddin, A. A. Khan, A. J. Haig, M. K. Uddin. Presentation of frozen shoulder among diabetic and non-diabetic patients. Journal of clinical orthopaedics and trauma. 2014;5(1):193-198. 